\title{
The Personal Sensor Network: a User-Centric Monitoring Solution
}

\author{
G. Giorgetti ${ }^{*}$, G. Manes \\ Dip. Elettronica e Telecom., Università \\ degli Studi di Firenze, ITALY \\ gianni.giorgetti@unifi.it \\ gianfranco.manes@unifi.it
}

\author{
J.H. Lewis, S.T. Mastroianni, \\ Embedded Systems Research, \\ Motorola Labs, Tempe, US \\ jonathan.lewis@motorola.com \\ s.mastroianni@motorola.com
}

\author{
S.K.S. Gupta \\ IMPACT Lab, School Comput. \& Info., \\ Arizona State University, Tempe, US \\ sandeep.gupta@asu.edu
}

\begin{abstract}
Server-based architectures used in traditional Wireless Sensor Network (WSN) applications are not suitable when the sensors are installed near the user and local access is desirable. We address this problem by proposing the Personal Sensor Network (PSN), a computer-less architecture that enables users to access the sensor data using their cell phones or any other Bluetooth enabled devices. The use of COTS hardware and widely available software resources results in a solution easy to implement and simple to interface with other WSNs.
\end{abstract}

\section{Categories and Subject Descriptors}

C.2.3 [Computer Communication Networks]: Network Operations - network management, network monitoring.

\section{General Terms}

Design, Experimentation.

\section{Keywords}

Wireless Sensor Network, IEEE 802.15.4, Bluetooth, Cell phone.

\section{INTRODUCTION}

In traditional WSN applications, the access to the network is mediated by a central server that collects the information before dispatching the results to the final user. While this model has been extensively developed and adopted in many remote-monitoring deployments, imposing a server-based solution seems unnatural when the sensors are installed near the user (e.g. ambient intelligence, smart-home) or even on his person (body area networks). In all these cases, allowing a direct interaction between the user and the nearby nodes would result in a more intuitive access to the sensor data, reduce the transmission delays, and in some cases, it would even eliminate the need to maintain a server and a remote link.

\footnotetext{
* The author is also affiliated with the Electrical Engineering Department at Arizona State University. He was an intern with Motorola Labs in the summer of 2006. His work is partly supported under contract IST508744-IP EU Integrated Project GoodFood.

Permission to make digital or hard copies of all or part of this work for personal or classroom use is granted without fee provided that copies are not made or distributed for profit or commercial advantage and that copies bear this notice and the full citation on the first page. To copy otherwise, to republish, to post on servers or to redistribute to lists, requires prior specific permission and/or a fee. BODYNETS 2007, June 11-13, Florence, Italy Copyright $($ C) 2007 ICST 978-963-06-2193-9 DOI 10.4108/bodynets.2007.156
}

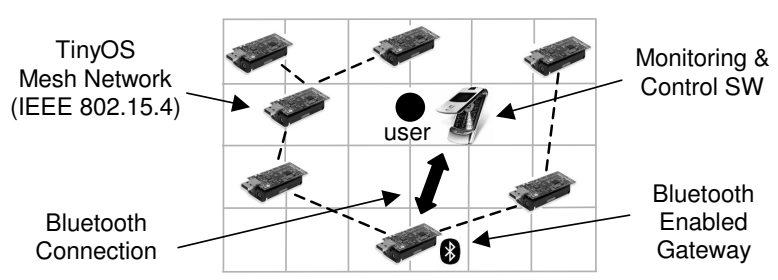

Figure 1. The Personal Sensor Network architecture.

We have explored the idea of a computer-less architecture by implementing a Personal Sensor Network (PSN) consisting of ready-to-use sensor nodes and a small Bluetooth gateway. The PSN is based on a user-centric model where the presence of a server is unnecessary because the user can access the network using the same cell phone (or any handheld device) that he usually carries around in his everyday activities. In addition, since all network devices are compact and lightweight, the PSN can be easily transported and used whenever a monitoring system is needed. For example, the safety of first responders to an incident scene could be increased by a PSN near, or on their body, that provides immediate access to the local sensor information.

In the next section we discuss the characteristics of the system implemented. The application should be considered as a proof-ofconcept solution aiming at demonstrating the synergy possible by combining established and rapidly maturing technologies (IEEE 802.15.4, Bluetooth and cell phones). While other phone-based approaches have been proposed in conjunction with custom solutions for healthcare applications [1][2], our approach entirely relies on COTS components and widely available software resources. The result is a solution that is easy to implement and simple to interface with existing sensor networks.

\section{SYSTEM OVERVIEW}

The PSN architecture (see Figure 1) is based on a TinyOS network consisting of IEEE 802.15.4 nodes and a gateway (GW) with support for Bluetooth (BT) connectivity. Using a cell phone, or any other BT enabled device, the user can connect to the GW and obtain real-time information from the sensors, send control commands or communicate specific information (e.g. a preference profile). Since the user can access the network while freely moving among the sensors, the phone not only replaces the PC, but also supports a more natural and intuitive interaction with the WSN. The BT interface on the gateway was designed to achieve a seamless integration between the phone and the sensor nodes. In pursuing this goal we were able to maintain the network structure unchanged to ensure compatibility with existing applications and 


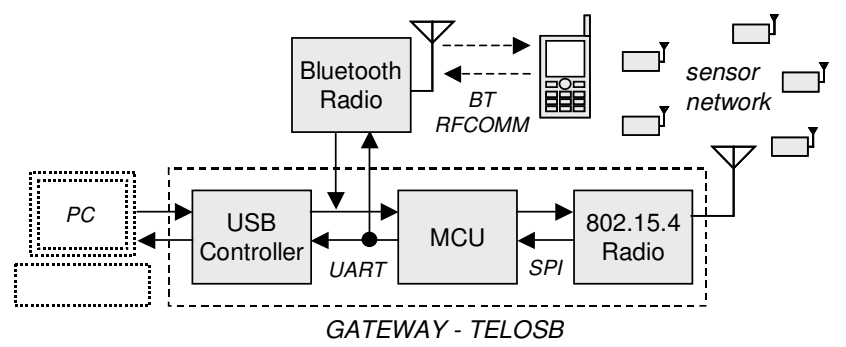

Figure 2. The gateway architecture.

allows the use of a PC when permanent data logging or complex computation is required.

\section{SENSOR NODES}

The PSN is based on TelosB boards, commercially available sensor nodes designed for low power operations and compatible with the IEEE 802.15.4/ZigBee standard. The nodes run a TinyOS based software that monitors a set of parameters relevant to the user: for example, in our application the PSN nodes are configured to report temperature, humidity, battery voltage and radio strength; some units were also interfaced to external $\mathrm{CO}_{2}$ sensors. The advantage of using TelosB nodes is that we can leverage TinyOS and its support for ad hoc multi-hop protocols (e.g. MultiHopLQI) to create a self-configuring network. Since the nodes are able to automatically select the most reliable route to the GW and the network topology dynamically adapts to changes in the system (e.g. nodes added or removed, radio link failures), deploying a PSN reduces to powering the nodes up and placing them close to the location to monitor.

\subsection{NETWORK GATEWAY}

One of the TelosB nodes serves as a gateway for the network and is usually connected to a PC through the USB interface. In the PSN architecture we extended the GW functionalities by adding a Bluetooth module that shares the same UART lines used to communicate with the PC (see Figure 2). By adopting this simple solution (only a pull-up resistor and a couple of diodes are used to isolate the TX lines), the gateway can serve a phone, a PC or both devices at the same time without modification to the software structure. Whenever a radio packet is received and the GW forwards it to the USB controller, a copy will also be received by the BT radio. Similarly, messages sent by the PC or the phone ${ }^{1}$ will be transparently forwarded to the IEEE 802.15.4 network.

The Bluetooth module (BlueRadios BR-C40A [3]) installed on the $\mathrm{GW}$ is a compact unit that offers a simple configuration interface based on AT-commands. The unit supports the Serial Port Profile and is configured to bridge the communication between the internal UART and the virtual port on the BT connection (RFCOMM). Although not all the functionalities are accessible (for example, it is not possible to create and advertise custom services through the Service Discovery Protocol), the available AT commands offer the possibility to tweak some lowlevel parameters useful to support battery-powered operations. In

${ }^{1}$ Message collisions can occur if the GW is connected to both a PC and a Phone, and both devices attempt to write on the UART lines at the same time. This does not pose a problem if commands to the WSN are issued sporadically.
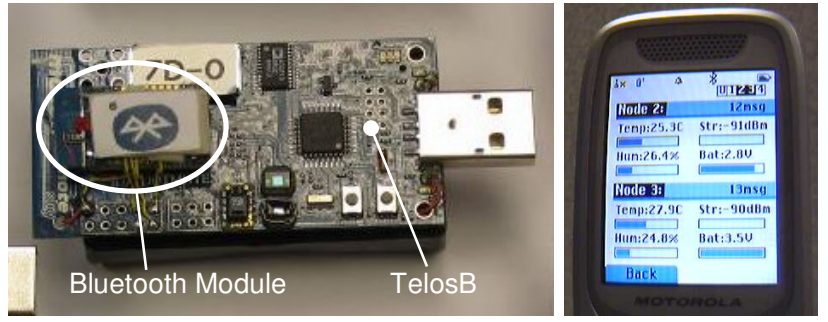

Figure 3. The Bluetooth gateway (left) and a detail of the phone interface (right).

particular, increasing the page scan interval and activating the sniff mode reduce the current consumption to $2 \mathrm{~mA}$ when the device is waiting for a connection; and, to $1 \mathrm{~mA}$ when the device is connected (but no data is exchanged).

\subsection{PHONE SOFTWARE}

The software running on the user phone is a MIDlet that can be installed on any device supporting JAVA Micro Edition and Bluetooth communication. The software is responsible for handling the connection with the Bluetooth radio on the GW, converting the raw sensor reading in engineering units and providing the user with an intuitive interface to access the sensor data and control commands (see Figure 3). Given the gateway architecture, the only requirement imposed on the software was to handle communication with the GW using the same packet structure adopted within the TinyOS network (in facts, the GW routes the packets between the WSN and the phone without modification). This feature was enabled with limited effort by utilizing part of the JAVA code distributed with TinyOS. Using a stripped-down version of the PC-based classes that implements packet handling over serial communication, we were able to sensibly reduce the prototyping time for the SW. Slightly modified versions of the phone software were created to run on a PDA and a Bluetooth Notebook.

\section{CONCLUSIONS}

The PSN demonstrates that with limited effort it's possible to seamlessly integrate mobile computing solutions with sensor network technology. In particular, the use of a cell phone is appealing since many people find this technology familiar and intuitive to use, a fact that was confirmed by the opinions of the users of our system. And, even though the aim of this work was to explore connectivity with nearby sensors, because many handheld devices have LAN capabilities as well as the WAN capabilities of the cellular network, each individual local cluster of PSN could also provide data to other centralized data servers.

\section{REFERENCES}

[1] S. Krco, Implementation solutions and issues in building a personal sensor network for health care monitoring, 4th Int. IEEE Conf. on Information Tech. and Applications in Biomedicine, pagg 350-353, 2003.

[2] L. Zhong, M. Sinclair, and R. Bittner, A Phone-Centered Body Sensor Network Platform: Cost, Energy Efficiency User Interface, BSN 2006.

[3] Blueradios BR-C40., http://www.blueradios.com 